\title{
Addendum: Effector memory T cell responses are associated with protection of rhesus monkeys from mucosal simian immunodeficiency virus challenge
}

Scott G Hansen, Cassandra Vieville, Nathan Whizin, Lia Coyne-Johnson, Don C Siess, Derek D Drummond, Alfred W Legasse, Michael K Axthelm, Kelli Oswald, Charles M Trubey, Michael Piatak Jr, Jeffrey D Lifson, Jay A Nelson, Michael A Jarvis \& Louis J Picker Nat. Med. 15, 293-299 (2009); published online 15 February 2009; corrected after print 6 April 2009; addendum published after print 7 November 2011

In Supplementary Figure 1 of our paper, the reference cited (Hel et al. in Vaccine 20, 3171-3186, 2002) and the description for the Retanef fusion gene expressed by RhCMV-Retanef were incorrect. The correct description for the fusion gene (here designated Retanef(int)) and citation are now contained in the Supplementary Information.

Retanef(int) is a fusion of simian immunodeficiency virus (SIV) rev (Met1-Leu19), int (Lys159-Ala293), full-length nef (starting at Ala4) and tat (Glu2-Arg82), mutagenized to decrease toxicity, essentially as previously described by Kulkarni et al. (Vaccine 29, 6742-6754, 2011). Table $\mathbf{1}$ shows a comparison between Retanef(Hel) (Vaccine 20, 3171-3186, 2002) and our Retanef(int) construct.

In Figure $4 \mathrm{~d}$ of our paper, the appearance of de novo $\mathrm{CD}^{+} \mathrm{T}$ cell responses to overlapping peptides comprising the full-length SIV pol protein in the four stringently protected RhCMV-SIV-vaccinated rhesus macaques after SIVmac239 challenge was used as evidence of occult SIV infection. Although the presence of a pol component-int(Lys159-Ala293) - within the RhCMV-Retanef(int) vector would bring this conclusion into question, several lines of evidence strongly support our original interpretation. First, as we initially indicated, two of the four protected rhesus macaques showed transient SIV viremia, and all four of these rhesus macaques showed the appearance of de novo CD8 ${ }^{+}$responses to peptides comprising full-length SIV vif (a protein not included in the vaccine). Second, peripheral blood mononuclear cells from a total of 23 RhCMV-Retanef(int)vaccinated and three SIV-infected rhesus macaques were found to be negative for both $\mathrm{CD} 4^{+}$and $\mathrm{CD} 8^{+} \mathrm{T}$ cell responses to peptide mixes comprising int (Lys159-Ala293), indicating this region is poorly immunogenic or nonimmunogenic for T cells in rhesus macaques. Finally, further analysis of cryopreserved T cells from the four protected monkeys in our study specifically shows the absence of CD8 ${ }^{+} \mathrm{T}$ cell responses to full-length pol, int(Lys159-Ala293) and the SIV rev/tat regions unique to the retanef(Hel) construct (and not in the RhCMV-retanef(int) vector administered to these rhesus macaques) prior to SIVmac239 challenge, as well as the appearance of such responses to full-length pol and the unique retanef(Hel) regions, but not the int(Lys159-Ala293), in all monkeys after SIV challenge (Fig. 1). Thus, challenge was associated with the induction of de novo CD8 ${ }^{+} \mathrm{T}$ cell responses to multiple distinct SIV sequences that were not included in the vaccine (that is, full-length vif, pol other than int(Lys159-Ala293), $\operatorname{rev}($ Leu20-Asp100) and tat(Arg83-Arg131)), consistent, as we originally suggested, with controlled SIV infection. Thus, none of the conclusions of our original report are affected by the use of RhCMV-Retanef(int) reported in this addendum.

Table 1 Comparison of amino acid sequences of Retanef(Hel) and Retanef(int)

\begin{tabular}{lllll}
\hline SIV regiona & Retanef(Hel) & Retanef(int) & $\begin{array}{l}\text { Retanef(Hel) } \\
\text { unique region }\end{array}$ & $\begin{array}{l}\text { Retanef(int) } \\
\text { unique region }\end{array}$ \\
\hline rev (mac251) & Met1-Asp100 & Met1-Leu19 & Leu20-Asp100 & - \\
nef (mac239) & His139-Glu241 & Ala4-Arg263 & - & Glu242-Arg263 \\
& + & & & \\
tat (mac251) & Ala4-Leu146 & & Arg83-Arg131 & - \\
& + & Glu2-Arg82 & & \\
int (mac239) & Glu2-Arg82 & & & Lys159-Ala293 \\
\hline
\end{tabular}

${ }^{a}$ Amino acid designation based on functionally inactivated SIV open reading frames, as described by Hel et al. and Kulkarni et al.

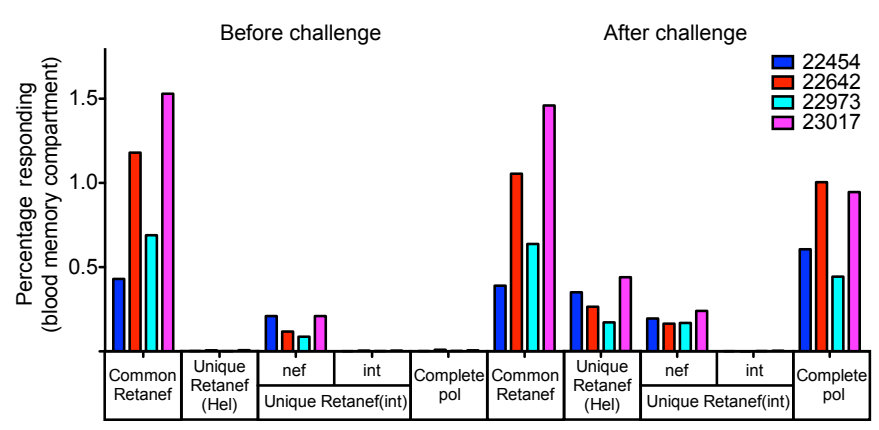

Figure 1 Immunogenicity profiles of RhCMV-retanef(int)-vaccinated controllers before and after SIV challenge. Flow cytometric intracellular cytokine analysis (FCICA) (Nat. Med. 15, 293-299, 2009) of peripheralblood $\mathrm{CD} 8^{+} \mathrm{T}$ cells responding to 15 -mer overlapping peptides comprising amino acid sequences common or unique to Retanef(Hel) and Retanef(int) in the four RhCMV-retanef(int)-vaccinated, SIV controllers we initially reported before and after SIV challenge. The percentage of responding SIV antigen-specific CD8 ${ }^{+} \mathrm{T}$ cells (producing tumor necrosis factor- $\alpha$, interferon- $\gamma$ or both) within the overall memory subset is shown. Responses before challenge were restricted to sequences included in the RhCMVretanef(int) vector, whereas after challenge responses to sequences that are only expressed by the SIV challenge virus are identified. The only exception to this pattern is the int(Lys159-Ala293) sequence, which, though included in the RhCMV-retanef(int) vector and in SIV itself, is nonimmunogenic for $\mathrm{T}$ cells both before and after challenge. 